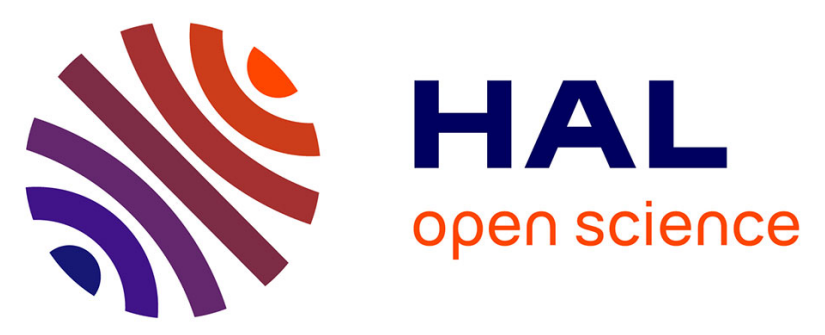

\title{
Impact of Singly Occupied Molecular Orbital energy on the n-doping efficiency of benzimidazole-derivatives
}

\author{
Sergi Riera-Galindo, Alessio Orbelli Biroli, Alessandra Forni, Yuttapoom \\ Puttisong, Francesca Tessore, Maddalena Pizzotti, Eleni Pavlopoulou, \\ Eduardo Solano, Suhao Wang, Gang Wang, et al.
}

\section{To cite this version:}

Sergi Riera-Galindo, Alessio Orbelli Biroli, Alessandra Forni, Yuttapoom Puttisong, Francesca Tessore, et al.. Impact of Singly Occupied Molecular Orbital energy on the n-doping efficiency of benzimidazole-derivatives. ACS Applied Materials \& Interfaces, 2019, 11 (41), pp.37981-37990. 10.1021/acsami.9b12441 . hal-02888890

\section{HAL Id: hal-02888890 https://hal.science/hal-02888890}

Submitted on 3 Jul 2020

HAL is a multi-disciplinary open access archive for the deposit and dissemination of scientific research documents, whether they are published or not. The documents may come from teaching and research institutions in France or abroad, or from public or private research centers.
L'archive ouverte pluridisciplinaire HAL, est destinée au dépôt et à la diffusion de documents scientifiques de niveau recherche, publiés ou non, émanant des établissements d'enseignement et de recherche français ou étrangers, des laboratoires publics ou privés. 


\title{
Impact of Singly Occupied Molecular Orbital energy on the $n$-doping efficiency of benzimidazole-derivatives
}

\author{
Sergi Riera-Galindo, ${ }^{\dagger}$ Alessio Orbelli Biroli, $\neq$ Alessandra Forni, $\neq$ Yuttapoom Puttisong,§ Francesca \\ Tessore," Maddalena Pizzotti," Eleni Pavlopoulou, ${ }^{\perp}$ Eduardo Solano,\# Suhao Wang, ${ }^{\dagger}$ Gang Wang, ${ }^{\dagger}$ \\ Tero-Petri Ruoko, ${ }^{\dagger}$ Weimin M. Chen,§ Martijn Kemerink, $₫$ Magnus Berggren, ${ }^{\dagger}$ Gabriele di Carlo,"** \\ Simone Fabiano ${ }^{* *}$
}

${ }^{\dagger}$ Laboratory of Organic Electronics, Department of Science and Technology, Linköping University, SE-60174 Norrköping, Sweden.

*Istituto di Scienze e Tecnologie Molecolari del CNR (CNR-ISTM), SmartMatLab Centre, via C. Golgi 19, 20133 Milano, Italy.

§Department of Physics, Chemistry and Biology (IFM), Linköping University, SE-58183 Linköping, Sweden. "Dipartimento di Chimica, Unitá di Ricerca dell'INSTM, Università degli Studi di Milano, via C. Golgi 19, 20133 Milano, Italy.

${ }^{\perp}$ Laboratoire de Chimie des Polymères Organiques (LCPO - UMR 5629), Bordeaux INP, Université de Bordeaux, CNRS, 16 Av. Pey-Berland, 33607, Pessac, France.

\#NCD-SWEET beamline, ALBA Synchrotron Light Source, Carrer de la Llum 2-26, 08290 Cerdanyola del Vallès, Spain.

ॠComplex Materials and Devices, Department of Physics, Chemistry and Biology (IFM), Linköping University, SE-

58183 Linköping, Sweden.

\begin{abstract}
We investigated the impact of Singly Occupied Molecular Orbital (SOMO) energy on the n-doping efficiency of benzimidazole-derivatives. By designing and synthesizing a series of new air-stable benzimidazole-based dopants with different SOMO energy levels, we demonstrated that an increase of the dopant SOMO energy by only $\sim 0.3 \mathrm{eV}$ enhances the electrical conductivity of a benchmark electron-transporting naphthalenediimide-bithiophene polymer by more than one order of magnitude. By combining electrical, X-ray diffraction, and electron paramagnetic resonance measurements with density functional theory calculations and analytical transport simulations, we quantitatively characterized the conductivity, Seebeck coefficient, spin density, crystallinity of the doped polymer as a function of the dopant SOMO energy. Our findings strongly indicate that charge and energy transport are dominated by the (relative) position of the SOMO level, whereas morphological differences appear to play a lesser role. These results set molecular-design guidelines for next-generation ntype dopants.
\end{abstract}

\section{INTRODUCTION}

Thanks to their versatile chemical synthesis, inexpensive solution processability and unique mechanical flexibility, ${ }^{1}$ $\pi$-conjugated polymers are finding application in a vast variety of present and future low-cost and distributed technologies, including organic solar cells (OSCs), ${ }^{2-4}$ organic light emitting diodes (OLEDs), ${ }^{3}$ organic thermoelectrics (OTEs), ${ }^{5-9}$ batteries and supercapacitors. ${ }^{10,11}$ The performance of these organic electronic devices strongly depends on the charge transport properties of the semiconductor active layers. The conductivity of several solution processable conjugated polymers, and therefore the performance of many of the corresponding devices can be enhanced by molecular doping. This can be achieved by either oxidizing (p-doping) or reducing ( $n$-doping) the $\pi$ conjugated semiconductor with a dopant, a strategy that increases the charge-carrier concentration, and thereby the electrical conductivity $(\sigma)$. For practical applications, both hole-transporting (p-type) and electron-transporting (n-type) conducting polymers are usually required. However, unlike p-doped polymers that exhibit $\sigma$ typically larger than $1000 \mathrm{~S} \mathrm{~cm}^{-1}, 12-15 \mathrm{n}$-doped polymers are yet at an early stage of development with conductivities only recently passing $1 \mathrm{~S} \mathrm{~cm}^{-1}$.16-19

The first dopants reported for n-type organic semiconductors were metal complexes and inorganic salts deposited by thermal evaporation, a process that is incompatible with high throughput, large area device manufacturing. ${ }^{20-24}$ Solution-processable aromatic molecules, such as charge transfer complexes, ${ }^{25}$ have also been used as n-type dopants, but they are unstable in air. The use of air-stable precursors, which are then converted by heat or light into the reducing agent, allows to overcome the air instability issue. ${ }^{26,27}$ The most notable example of solutionprocessable and air-stable dopant is the 4-(1,3-dimethyl- 
2,3-dihydro-1H-benzoimidazol-2-yl)-N,N-dimethylaniline (N-DMBI). ${ }^{28}$ It represents a class benzimidazole-based dopants that can effectively n-dope semiconducting polymers $^{29}$ and small molecules ${ }^{30}$ via hydride ${ }^{31}$ or electron ${ }^{28,32-}$ 34 transfer, yielding high electron conductivities. Schlitz et al., for example, used N-DMBI to dope the high electron mobility polymer poly(N,N'-bis-2octyld odecylnaphthalene-1,4,5,8-bis-dicarboximide-2,6diyl-alt-5,5'-2,2'-bithiophene) P(NDI20D-T2), obtaining an optimized electrical conductivity of $\sim 0.001 \mathrm{~S} / \mathrm{cm} .{ }^{29}$ The low conductivity was attributed to the strong charge carrier localization on the polymer backbone ${ }^{35}$ and to the limited solubility of benzimidazole-based dopants in the host polymer, which restricts the doping efficiency to only $\sim 1 \%$. $^{36}$ Following that study, many reports have focused on improving the miscibility of the dopant, by tailoring either the host polymer backbone ${ }^{37-42}$ or the dopant molecular structure. ${ }^{36}$ Despite the progress in this field, the role of the benzimidazole-based dopant energetics on the doping efficiency remains poorly understood.

Here, we investigate the influence of the Singly Occupied Molecular Orbital (SOMO) energy on the doping efficiency of benzimidazole-derivatives, with P(NDI20D-T2) used as a benchmark n-type host polymer. We designed and synthesized a series of benzimidazoles in which the substitutes on the benzo-ring and 2-phenyl ring have been systematically varied, in order to adjust the energy of the SOMO level (Figure 1). We observed that a variation in the SOMO energy of only $\sim 0.3 \mathrm{eV}$ induces an increase in the electrical conductivity of the doped P(NDI20D-T2) films by more than one order of magnitude. By combining electrical, X-ray diffraction, and electron paramagnetic resonance measurements with density functional theory calculations and analytical transport simulations, we quantitatively characterized the electrical conductivity, Seebeck coefficient, spin density, crystallinity of P(NDI20D-T2) films doped with the benzimidazole-derivatives. Complementing recent works focusing on the guest dopant/host polymer miscibility, we show here that the SOMO energy has a strong effect on the doping efficiency of benzimidazolederivatives and needs to be taken into consideration when synthesizing new benzimidazole-based dopants.

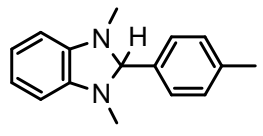

$1 \mathrm{H}$

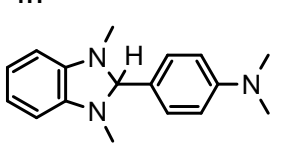

4H (N-DMBI)

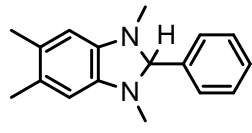

$2 \mathrm{H}$

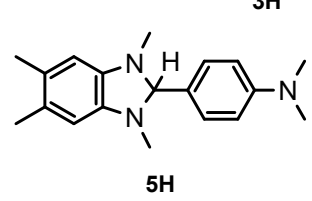

Figure 1. Molecular structures of the benzimidazoline-based dopants investigated in this study.

\section{EXPERIMENTAL SECTION}

Dopant synthesis. All dopants were synthesized following the procedures reported in the Supporting Information. All reagents and solvents involved in the synthesis were purchased from Sigma Aldrich and used as received, except THF (freshly distilled from $\mathrm{Na}$ /benzophenone un- der nitrogen atmosphere). Glassware has been flame-dried under vacuum before use when necessary. ${ }^{1} \mathrm{H}-\mathrm{NMR}$ and ${ }^{13} \mathrm{C}$-NMR spectra were recorded on a Bruker Avance DRX400 and on a Bruker AMX 300 in $\mathrm{CDCl}_{3}$ or DMSO- $d_{6}$ as solvents. Mass spectra were obtained with a VG Autospec M246 magnetic mass spectrometer with a LSIMS ionic source. Elemental analysis was carried out with a PerkinElmer CHN 2400 instrument.

Calorimetric and Thermogravimetric Measurements. Differential scanning calorimetry (DSC) measurements of pristine dopants were performed on Al-crucibles $(40 \mu \mathrm{l})$ under $\mathrm{N}_{2}$ at $10 \mathrm{~K} \mathrm{~min}^{-1}$ with a Mettler Toledo DSC 3 instrument equipped with a Huber TC100-MT cooling system. Thermogravimetry measurements were performed on alumina crucibles $(70 \mu \mathrm{l})$ under $\mathrm{N}_{2}$ at $10 \mathrm{~K} \mathrm{~min}^{-1}$ with a Mettler Toledo TGA/DSC $3^{+}$. Both TGA and DSC analysis were processed by STAR ${ }^{\mathrm{e}}$ software.

Electrochemical characterization. The voltammetric studies were performed in a $4 \mathrm{~cm}^{3}$ cell, on ca. $2.5 \times 10^{-3} \mathrm{M}$ solutions in acetonitrile (Aldrich, 99.8\%) with $0.1 \mathrm{M}$ tetrabutylammonium perchlorate (TBAP, Fluka) as the supporting electrolyte. The solutions were de-aerated by $\mathrm{N}_{2}$ bubbling. The ohmic drop was compensated by the positive feedback technique. ${ }^{43}$ The experiments were carried out using an AUTOLAB PGSTAT potentiostat (EcoChemie, The Netherlands) run by a PC with GPES software. Cyclic voltammetry (CV) investigations were carried out at scan rates typically ranging from 0.05 to $2 \mathrm{Vs}^{-1}$, with ohmic drop compensation. Differential pulse voltammetry (DPV) curves were recorded with a step potential of $5 \mathrm{mV}$ and a modulation amplitude of $50 \mathrm{mV}$. The working electrode was a glassy carbon one (AMEL, diameter $=1.5 \mathrm{~mm}$ ) cleaned by synthetic diamond powder (Aldrich, diameter = $1 \mu \mathrm{m}$ ) on a wet cloth (STRUERS DP-NAP); the counter electrode was a platinum wire. The operating reference electrode was an aqueous saturated calomel electrode, but the potentials were ultimately referred to the $\mathrm{Fc}^{+} / \mathrm{Fc}$ (ferrocinium/ferrocene) couple. ${ }^{44,45}$ To prevent water and chloride leakage into the working solution a compartment filled with the operating medium and ending with a porous frit was interposed between the reference electrode and the cell.

Film preparation and doping. Polymer films were prepared inside a nitrogen-filled glovebox. P(NDI2OD-T2) ActivInk N2200 (Mn = 29.7 kDa) was purchased from Flexterra. P(NDI20D-T2) (5 mg/ml) and benzimidazole dopants were dissolved in $\mathrm{C}_{6} \mathrm{H}_{4} \mathrm{Cl}_{2}$. All solutions were stirred at $60{ }^{\circ} \mathrm{C}$ overnight to ensure complete dissolution. Later, appropriate amounts of each dopant solution were added to the P(NDI2OD-T2) solution and stirred for at least 1 hour. Glass substrates were cleaned sequentially in ultrasonic bath with acetone and isopropanol for $5 \mathrm{~min}$ each and dried by nitrogen flow. $3 \mathrm{~nm} \mathrm{Cr}$ and $20 \mathrm{~nm} \mathrm{Au}$ were deposited by thermal evaporation on glass substrates through shadow mask to define a two-probe geometry. Doped solution at each molar ratio were spin-coated (1000 rpm for $30 \mathrm{~s}$ ) on glass with pre-patterned electrical contacts, and subsequently thermally annealed at $150{ }^{\circ} \mathrm{C}$ under nitrogen atmosphere for $30 \mathrm{~min}$.

Optical and Electrical Characterization. Absorption measurements of thin films were performed in nitrogen 
and at room temperature by using an UV-vis-NIR spectrophotometer (PerkinElmer Lambda 900). Electrical conductivity was measured in a $\mathrm{N}_{2}$-filled glovebox using a Keithley 4200-SCS semiconductor parameter analyzer and determined from the slope $d I /\left.d V\right|_{V=0}$ for multiple devices per sample. The channel dimensions were $\mathrm{L} / \mathrm{W}=30 \mu \mathrm{m} / 1000$ $\mu \mathrm{m}$. The Seebeck coefficients of the doped films were also measured under nitrogen atmosphere. The temperature gradient $(\Delta \mathrm{T})$ across the sample was fixed by two Peltier modules, and the thermovoltage $(\Delta \mathrm{V})$ was probed between two separate electrodes (L/W $=0.5 \mathrm{~mm} / 15 \mathrm{~mm}$ ). The Seebeck coefficient was obtained from the slope of $\Delta \mathrm{V}$ measured at five different $\Delta \mathrm{T}$ values.

EPR and KP Measurements. Quantitative electron paramagnetic resonance (EPR) experiments were performed at the Swedish Interdisciplinary Magnetic Resonance Centre (SIMARC) at Linköping University, using a Bruker Elexsys E500 spectrometer operating at about 9.8 $\mathrm{GHz}$ (X-band). EPR spectra were recorded in dark at room temperature. A quantitative spin counting was calibrated via a standard sample. All EPR spectra were normalized by an effective detection volume of the samples. The work function of doped films, deposited under the same conditions as for conductivity and Seebeck coefficient samples on a 40-nm gold layer, were determined by Kelvin Probe (SKP5050, KP Technology Ltd). Freshly exfoliated highly ordered pyrolytic graphite (HOPG) was used as the reference sample $(\mathrm{WF}=-4.6 \mathrm{eV})$.

Thin-Film Morphology Characterization. Atomic force microscopy (AFM) (Dimension 3100 microscope with Nanoscope IIIa controller; Digital Instruments) was operated in tapping mode to characterize the surface morphologies and film thickness. Grazing Incidence Wide Angle X-ray Scattering (GIWAXS) experiments were performed at the NCD-SWEET beamline of the ALBA Synchrotron, located in Cerdanyola del Vallès, Spain. The wavelength of the X-rays, $\lambda$, was $0.9998 \AA$ (12.4 keV), the sample-to-detector distance was $131.3 \mathrm{~mm}$ and the angle of incidence, $\alpha_{i}, 0.15^{\circ}$. The diffracted intensity was recorded using a Rayonix LX255-HS detector, which consists of a $1920 \times 5760(\mathrm{H} \times \mathrm{V})$ pixel array with a pixel size of $44 \times 44$ $\mu \mathrm{m}^{2}$. Data were normalized by the incident photon flux and the acquisition time. Flat field, polarization, solid angle and efficiency corrections were subsequently applied to the 2D GIWAXS images. The scattering vector $q$ was defined with respect to the center of the incident beam and has a magnitude of $q=(4 \pi / \lambda) \sin (\theta)$, where $2 \theta$ is the Bragg reflection angle. The wedge-shaped corrected images are presented in this manuscript, where $q_{x y}$ and $q_{z}$ are the in-plane and near out-of-plane scattering vectors, respectively. The scattering vectors are defined as follows: $q_{\mathrm{x}}=(2 \pi / \lambda)\left(\cos \left(2 \theta_{f}\right) \cos \left(\alpha_{f}\right)-\cos \left(\alpha_{i}\right)\right)$,

$q_{\mathrm{y}}=(2 \pi / \lambda)\left(\sin \left(2 \theta_{f}\right) \cos \left(\alpha_{f}\right)\right), \quad q_{z}=(2 \pi / \lambda)\left(\sin \left(\alpha_{f}\right)+\sin \left(\alpha_{i}\right)\right)$, $q_{x y}^{2}=q_{x}^{2}+q_{y}^{2}$ where $\alpha_{f}$ is the exit angle in the vertical direction and $2 \theta_{f}$ is the in-plane scattering angle. ${ }^{46}$ Therefore, the scattering vector is calculated as follows $q^{2}=$ $q_{x y}^{2}+q_{z}^{2}$.

DFT Calculations. Theoretical calculations have been performed on the benzimidazole-based compounds in their reduced neutral $(\mathbf{1 H}-\mathbf{5 H})$, neutral radical $\left(\mathbf{1}^{\bullet}-\mathbf{5}^{\bullet}\right)$ and oxidized ionic $\left(\mathbf{1}^{+}-\mathbf{5}^{+}\right)$forms to get HOMOs, SOMOs and
LUMOs, respectively. Calculations have been carried out at the (U)B3LYP/6-311++G(d,p) level of theory using the Gaussian 16 program package (Revision A.03). ${ }^{47}$ The geometry of all compounds has been optimized both in vacuum and in acetonitrile starting from the X-ray structure of the triflate salt of $\mathbf{1}^{+}$and introducing the appropriate substitutions. The effect of solvent was taken into account through the PCM approach based on the integral equation formalism model (IEFPCM). ${ }^{48}$

\section{RESULTS AND DISCUSSION}

Design and synthesis of benzimidazoline-based dopants. In 2008, Cheng et al. reported a comparative study on the electron, hydride and hydrogen transfer ability of several heterocyclic organic hydrides. ${ }^{28}$ Among all the surveyed hydrides, benzimidazolines (BHs) resulted the strongest reductants and the authors evidenced increased oxidation standard potentials (more negative) when electron-donating groups were introduced on the 2phenyl ring and an even larger effect when the substituents were positioned at the benzo-ring. Inspired by this work we rationally designed and synthesized three BHs (1H-3H), introducing electron-donating methyl groups on the benzo-ring and/or on the 2-phenyl ring (Figure 1). The benchmark benzimidazoline N-DMBI (4H), carrying a strong donating dimethylamino group on the 2-phenyl ring, was synthesized for comparison and its analogous (5H), bearing 2 further methyl donating moieties on the benzo-ring, was designed and synthesized in order to boost the doping efficiency and to prove the robustness of our approach.

Although several synthetic routes have been reported in the literature as general methods to get benzimidazole compounds, some procedures required a specific customization due to the presence of sensitive moieties on the starting materials or intermediates. The synthetic pathways of BHs were finely-tuned to obtain the desired dopants in few and trivial steps (Figure 2). A first oxidative cyclocondensation from commercially available aryl diamine and aryl aldehydes was drawn to give the benzimidazole core (B). When $p$-tolualdehyde and benzaldehyde were used as the starting materials, the reaction effectively took place in ethanol in the presence of hypervalent iodine reactant activated in situ by oxidation of $\mathrm{Bu}_{4} \mathrm{NI}$ with $\mathrm{H}_{2} \mathrm{O}_{2}$ as reported by Zhu et al. ${ }^{49}$ In contrast, low reaction yields were obtained with 4-(dimethylamino)benzaldehyde as the starting material. Thus, to boost the reaction yield, we explored an alternative cyclocondensation route previously reported to synthesize benzothiazole derivatives. ${ }^{50,51}$ As a result the precursors were promptly converted in the corresponding intermediate $\mathrm{B}$ with reasonable yields in DMSO, which acts simultaneously as solvent and oxidant species. 


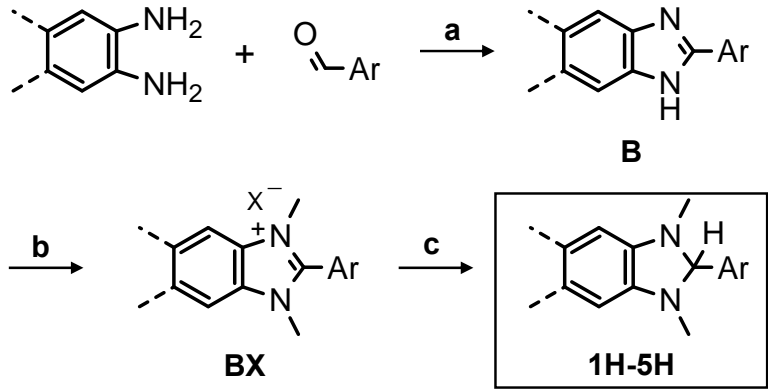

Figure 2. General synthesis of benzimidazolines: a) $\mathrm{Bu}_{4} \mathrm{NI}$, $\mathrm{H}_{2} \mathrm{O}_{2}$, EtOH, rt, o.n. or DMSO, $200{ }^{\circ} \mathrm{C}, 30 \mathrm{~min}$; b) $\mathrm{CH}_{3} \mathrm{I}$, tBuOK, THF, $60{ }^{\circ} \mathrm{C}, 48 \mathrm{~h} / \mathrm{AgOTf}, \mathrm{MeOH}, \mathrm{rt}$; c) $\mathrm{NaBH}_{4}, \mathrm{MeOH}, 0^{\circ} \mathrm{C}, 1 \mathrm{~h}$.

The following alkylation step was optimized to favour the formation of dimethyl benzimidazolium salts (B-X in Figure 2) in a one-pot strategy. ${ }^{52}$ The reaction smoothly proceeded at $60{ }^{\circ} \mathrm{C}$ in THF with stoichiometric amount of tBuOK and an excess of methyl-iodide to promote the simultaneous methylation of the two benzimidazole nitrogens to yield the desired B-I salts. The iodide counterion was also replaced with triflate to give B-OTf to prevent any influence of iodine on the redox potentials during the electrochemical measurements. The anion exchange step proceeded almost quantitatively by performing the reaction at room temperature in methanol with silver trifluoromethanesulfonate. The final reductive step successfully converted the benzimidazolium salts in the corresponding benzimidazolines with excess of $\mathrm{NaBH}_{4}{ }^{53}$ It is worth mentioning that while $\mathbf{1 H}, \mathbf{2} \mathbf{H}$, and $\mathbf{3 H}$ derivatives have easily been obtained by both iodide and triflate salt precursors, $\mathbf{4 H}$ and $\mathbf{5 H}$ derivatives have been formed with appreciable yield only from reduction of triflate precursors. Eventually the optimized synthetic approach provided a rapid, effective and scalable pathway to prepare the benzimidazolinebased compounds. All synthesized dopants were chemically characterized (Figures S1-S5) and their physical and electrochemical properties investigated.

Dopants characterization. In order to gain insight into the thermal stability of the synthesized dopants, we performed DSC and TGA measurements in the temperature range $30-300{ }^{\circ} \mathrm{C}\left(10^{\circ} \mathrm{C} / \mathrm{min}\right)$ under nitrogen atmosphere. The presence of two methyl groups on the benzo-ring greatly impacts the dopant melting points (Figures S6S10). Indeed $\mathbf{2} \mathbf{H}, \mathbf{3 H}$ and $\mathbf{5 H}$ derivates show melting temperatures over $160{ }^{\circ} \mathrm{C}$, much higher than those of $\mathbf{1 H}$ and 4H derivates (ca. $100-120^{\circ} \mathrm{C}$ ) which lack the methyl groups. TGA analysis show that the onset temperature for degradation of all dopants lies at around $200{ }^{\circ} \mathrm{C}$. By monitoring the weight losses, only $25 \%$ of the initial mass of $\mathbf{1 H}$ remains at $250{ }^{\circ} \mathrm{C}$, while $60 \%$ and $64 \%$ can be recovered from $\mathbf{2 H}$ and $\mathbf{3 H}$, respectively. A slighter difference is observed comparing $\mathbf{4 H}$ and $\mathbf{5 H}$ which maintain $86 \%$ and $92 \%$ of the initial weight. Overall, the collected data suggest a positive effect on the thermal stability by introducing the methyl substituents at the benzo-ring.

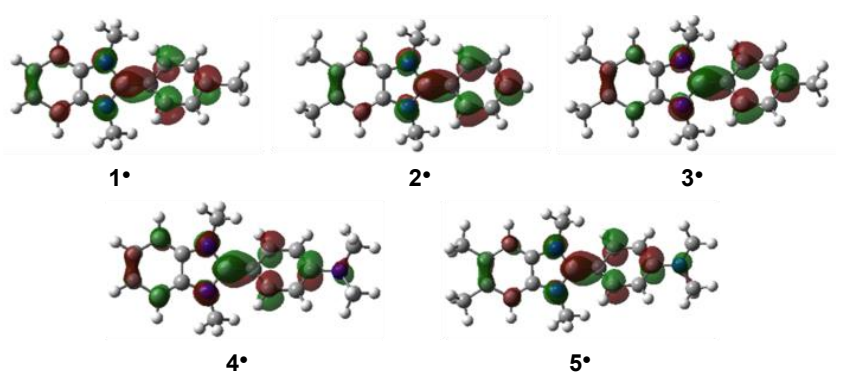

Figure 3. Plot of the SOMO of all synthesized dopants with isosurface value 0.04 a.u..

The dopant energetics were determined by a combination of electrochemical measurements and DFT calculations. Cyclic voltammetry (CV) and differential pulse voltammetry (DPV) measurements were performed on the benzimidazoline compounds, and their corresponding benzimidazolium triflate salt precursors (see Supporting Information for further details). The electrochemically derived highest occupied molecular orbital (HOMO) and lowest unoccupied molecular orbital (LUMO) energies were then compared to the ones estimated by DFT. The results are summarized in Tables S1-S2. Although the DFTcomputed HOMO levels show lower energies compared to the CV-derived ones, the trends are in good agreement and both methods confirm the destabilizing effect of the benzoring modification on the HOMO energies (Figures S11-S15 and Tables S1-S2). This is consistent with the HOMO being predominantly localized on the benzo-ring (Figure S16). A similar trend can be observed for $\mathbf{4 H}$ and $\mathbf{5 H}$, despite the electrochemical oxidation potentials being affected by the dimethylamino donor group on the 2-phenyl ring. Such a donor group slightly influences the CV-derived HOMO levels, a result nicely reproduced by DFT. Dopant $\mathbf{5 H}$, carrying both methyl substituents on the benzo-ring and the strong donor on 2-phenyl ring, shows the highest lying HOMO energy in the series from both theoretical calculations and electrochemical measurements. Thus, the measured HOMO levels vary in the dopant series as $\mathbf{1 H}(-4.68 \mathrm{eV})<\mathbf{4 H}(-$ $4.62 \mathrm{eV})<\mathbf{2} \mathbf{H}(-4.55 \mathrm{eV})<\mathbf{3} \mathbf{H}(-4.54 \mathrm{eV})<\mathbf{5 H}(-4.49 \mathrm{eV})$, with an average value at $-4.68 \mathrm{eV}$. This value is much lower than the LUMO of P(NDI20D-T2) which is around -3.90 $\mathrm{eV},{ }^{54}$ such that no electron transfer can spontaneously occur between the guest dopant and the host polymer.

It is known that the doping ability of benzimidazolebased compounds arises from activation of the pristine dopants into highly energetic radicals. ${ }^{30,55}$ For this reason, we computed the SOMO energy levels of the radical species $\left(\mathbf{1}^{\bullet}-\mathbf{5}^{\bullet}\right)$. The computed molecular orbital (MO) energy levels are reported in Table S2 and plotted in Figure 3 with isosurface value 0.04 a.u.. Unlike the HOMO levels, both the DPV-derived and DFT-computed LUMOs as well as the DFT-computed SOMOs increase linearly (less negative) in the dopant series $\mathbf{1 H}(-3.02 \mathrm{eV})<\mathbf{2 H}(-2.99 \mathrm{eV})<\mathbf{3 H}(-$ $2.91 \mathrm{eV})<\mathbf{4 H}(-2.82 \mathrm{eV})<\mathbf{5 H}(-2.73 \mathrm{eV})$. The methyl groups have a stronger effect on the SOMO (and LUMO) destabilization when they are on the benzo-ring rather than on the 2-phenyl ring, as is evident by comparing the energies of the singly occupied MO levels of $\mathbf{1 H}, \mathbf{2 H}$ and $\mathbf{3 H}$ (Figure 3 and Tables S1-S2). On the other hand, when a dimethylamino group is positioned on the 2-phenyl ring, as 
in the case of $\mathbf{4 H}$ and $\mathbf{5 H}$, its strong electron donating ability induces the SOMO to destabilize to a greater extent. As a consequence, the SOMO (and LUMO) level of $\mathbf{4 H}$ show higher energy than that of $\mathbf{2 H}$ bearing a methyl-substituted benzo-ring. Finally, it is worth noting that the simultaneous introduction of methyl groups on benzo-ring and of dimethylamino donor on 2-phenyl ring, as for $\mathbf{5 H}$, has a synergistic effect which leads to even higher SOMO and LUMO energy levels.

Electrical characterization. The electrical conductivity of doped P(NDI2OD-T2) thin films is reported in Figure 4a as a function of the dopant molar concentration. In its undoped pristine state, P(NDI20D-T2) has an electrical conductivity as low as $\sim 4 \times 10^{-7} \mathrm{~S} \mathrm{~cm}^{-1}$. After doping, the conductivity dramatically increases by several orders of magnitude with respect to the pristine P(NDI20D-T2) films, regardless of the dopant used. Remarkably, for dopant concentrations $\leq 25 \mathrm{~mol} \%$, we found that the conductivity of doped P(NDI2OD-T2) increases exponentially towards shallower (less negative) SOMO energy of the molecular dopant by $\sim 0.30 \mathrm{eV}$ (Figure $4 \mathrm{~b}$ ). The derivative $\mathbf{5 H}$, having a DFT-calculated $E_{\text {Sомо }}=-2.73 \mathrm{eV}$, yields the highest electrical conductivity $\left(\sim 3 \times 10^{-3} \mathrm{~S} \mathrm{~cm}^{-1}\right)$ at $25 \mathrm{~mol} \%$, which is more than one order of magnitude higher than that achieved by $\mathbf{1 H}\left(E_{\text {Sомо }}=-3.02 \mathrm{eV}\right)$ at the same dopant concentration $\left(\sim 1.6 \times 10^{-4} \mathrm{~S} \mathrm{~cm}^{-1}\right)$. Notably, we observed that the maximum conductivity value shifts towards higher molar concentrations for dopant molecules having higher SOMO energy. For instance, 1H-doped P(NDI2OD-T2) reaches a maximum conductivity of $8.3 \times 10^{-4} \mathrm{~S} \mathrm{~cm}^{-1}$ at 100 mol\%, whereas only $15 \mathrm{~mol} \%$ of $\mathbf{5 H}$ is needed to get the same conductivity values. However, for dopant concentrations > $25 \mathrm{~mol} \%$ (Figure S17), the dependence of conductivity with the SOMO energy is lost, most likely due to segregation of the dopant (Figure S18). ${ }^{56,57}$ Note that the conductivity values and trends obtained for P(NDI20D-T2) doped with $\mathbf{4 H}$ (i.e., N-DMBI) are consistent with previous reports, ${ }^{29,39,58}$ assuring that the films investigated in this study are representative of the high-performance material.

We also measured the Seebeck coefficient $(S)$ of P(NDI20D-T2) films doped with the different benzimidazole-derivatives (Figure 4c). The negative sign of $S$ is consistent with electrons being the majority carrier. Note that the sign inversion observed for 5H-doped P(NDI2OD-T2) at $100 \mathrm{~mol} \%$ is most likely due to the high electrical resistance which changes dramatically while heating the substrate, thus introducing an error in the extraction of $S$. As expected, $S$ is reduced when the dopant concentration is increased, regardless of the dopant molecule. Interestingly, $S$ decreases towards higher lying SOMO energies at all molar concentrations. The Seebeck coefficients for dopant concentrations $\leq 25 \mathrm{~mol} \%$ are reported as function of the SOMO energy in Figure 4d ( $S$ for dopant concentrations > $25 \mathrm{~mol} \%$ are shown in Figure S19). $S$ reduces for less negative SOMO energy at all concentrations, and this effect is larger at higher dopant concentration. These tendencies are in agreement with the observed conductivity trends. The resulting power factor, defined as $\mathrm{PF}=S^{2} \sigma$, increases by more than 5 times when P(NDI2OD-T2) is doped with $15 \mathrm{~mol} \% \mathbf{5 H}\left(0.011 \mu \mathrm{W} \mathrm{m} \mathbf{K}^{-1} \mathrm{~K}^{-2}\right)$ as compared to $\mathbf{1 H}(0.002$ $\mu \mathrm{W} \quad \mathrm{m}^{-1} \quad \mathrm{~K}^{-2}, \quad$ Figure 

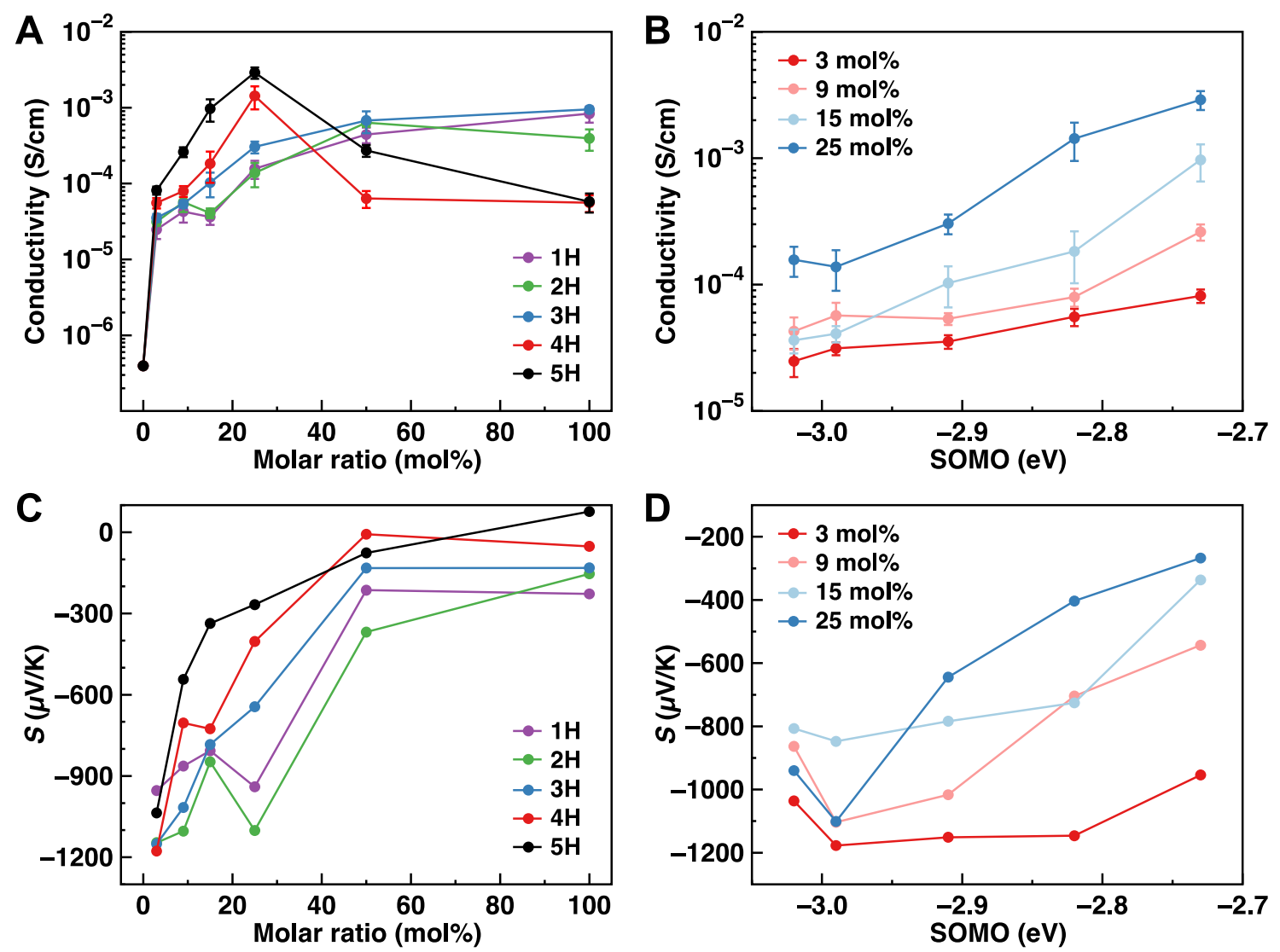

Figure 4. Electrical conductivity of doped P(NDI2OD-T2) as a function of dopant concentration (a) and as a function of SOMO energy of the dopant molecules (b). Seebeck coefficient of doped P(NDI2OD-T2) as a function of dopant concentration (c) and as a function of SOMO energy of the dopant molecules (d).

EPR Analysis. To shed light on the doping process, P(NDI20D-T2) films doped with the different benzimidazole-derivatives were investigated via electron paramagnetic resonance (EPR). EPR was performed at room temperature in an oxygen-free environment and the results, relative to a representative dopant concentration of $15 \mathrm{~mol} \%$, are reported in Figure 5. While pristine P(NDI2OD-T2) films are silent in EPR, doped P(NDI20D-T2) films show the presence of paramagnetic species with a characteristic electron g-factor of about 2.0021, regardless of the benzimidazole-derivative (Figure 5a). Consistent with the measured conductivity trends above, the EPR signal intensity increases towards shallower SOMO energies, indicating that $\mathbf{5 H}$-doped $\mathrm{P}(\mathrm{NDI} 2 \mathrm{OD}-\mathrm{T} 2)$ possesses a significantly larger amount of paramagnetic species compared to $\mathbf{1 H}$ doped P(NDI2OD-T2). Quantitative analysis, performed by normalizing the EPR signals over the measured sample volume, reveals that the spin density increases exponentially with higher lying SOMO energy, with 5H-doped $\mathrm{P}\left(\right.$ NDI20D-T2) possessing a spin density of $4.50 \times 10^{16}$ $\mathrm{mm}^{-3}$, which is about one order of magnitude above that of 1H-doped P(NDI2OD-T2) $\left(4.57 \times 10^{15} \mathrm{~mm}^{-3}\right)$. In addition to this observation, the spectral linewidth decreases going from $0.90 \mathrm{mT}$ to $0.42 \mathrm{mT}$ when P(NDI2OD-T2) is doped with $\mathbf{1 H}$ and $\mathbf{5 H}$, respectively (Figure $5 \mathrm{~b}$ ). The narrowing of the EPR linewidth is consistent with a higher charge density. ${ }^{54}$ We also confirmed this observation by means of Kelvin probe, which shows that the work function of doped P(NDI20D-T2) thin films decreases roughly linearly with higher lying SOMO energy (Figure S21), indicative of exponentially increasing doping levels (being the Fermi energy $E_{\mathrm{F}} \propto \ln \left(n / N_{0}\right)$, with $n / N_{0}$ the charge concentration). Furthermore, the absorbance difference between the doped and undoped P(NDI20D-T2) shows a tendency of higher absorbance for films doped with shallower SOMO energy dopants at $\sim 820 \mathrm{~nm}$ (Figure S22), which is consistent with the formation of a higher concentration of negative polarons. ${ }^{17}$
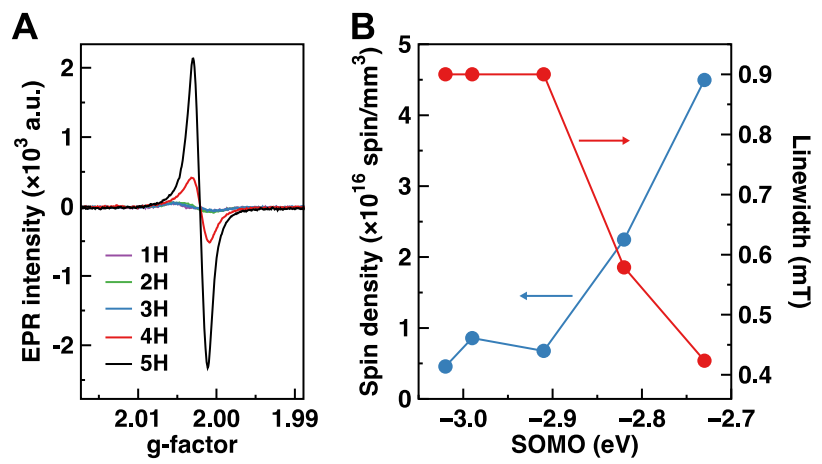

Figure 5. (a) EPR spectra (volume normalized) and (b) spin density and linewidth vs. SOMO energy of doped P(NDI2ODT2) at $15 \mathrm{~mol} \%$ dopant concentration.

Film Microstructure. Grazing incidence wide angle Xray scattering (GIWAXS) was performed to assess the impact of the different benzimidazole-derivatives on the sol- 
id-state microstructure of P(NDI2OD-T2). The recorded 2D diffraction patterns (Figure 6) were analyzed after background subtraction, wedge correction and scattering volume normalization, allowing for direct quantitative comparison between samples. The in-plane scattering data (Figure S23a) of the undoped P(NDI2OD-T2) films show reflections associated with the $(h 00)$ and $(00 l)$ families that, together with the presence of a significant (010) reflection in the out-of-plane scattering direction (Figure S23b), are indicative of a preferential face-on orientation of the polymer chains. The in-plane GIWAXS data show a main peak at $0.247 \AA^{-1}$, which corresponds to a lamellar (100) plane with a $d_{100}$ spacing in real space of $25.4 \AA$, in agreement with previous reports. ${ }^{59}$ The (200), (300) and (400) scattering peaks are also visible, attesting the high structural order of the polymeric films. Moreover, the (001) backbone reflection was found at $0.46 \AA^{-1}$, yielding a repeat unit length of $13.7 \AA$. The higher order (002) backbone reflection is also visible at the expected $\mathrm{q}_{\mathrm{z}}$ values. ${ }^{58,60}$ The presence of the $(00 I)$ planes for the in-plane data suggests that the polymer chains largely lie with their backbones parallel to the substrate. Besides the $(010)$ peak located at $1.61 \AA^{-1}$ ( $\pi-\pi$ stacking of $3.9 \AA$ ), the out-of-plane scattering data reported in Figure S23b also show the presence of strong $(h 00)$ reflections. This is indicative of the existence of well-ordered, edge-on oriented P(NDI2ODT2) lamellae. The out-of-plane (100) peak of the pristine film, located at $\mathrm{q}_{\mathrm{z}}=0.255 \AA^{-1}$, corresponds to a lamellar $\mathrm{d}$ spacing of $24.6 \AA$. This value is slightly smaller than the $\mathrm{d}_{100}$-spacing calculated from the in-plane data $(25.4 \AA)$, suggesting that the edge-on lamellae are closer-packed with respect to the face-on oriented lamellae, in agreement with previous reports. ${ }^{61}$

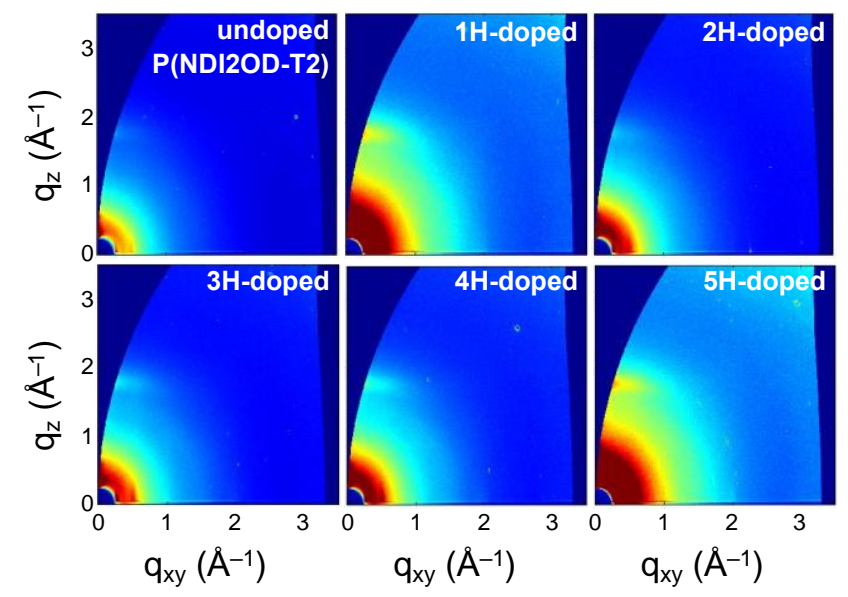

Figure 6. 2D GIWAXS patterns of undoped and doped P(NDI2OD-T2) at 15 mol\% dopant concentration.

Doped P(NDI2OD-T2) films, regardless of the used benzimidazole dopants, show the same sets of in-plane and out-of-plane reflections as the undoped film, suggesting that the overall crystallite orientation is not affected, i.e. edge-on and face-on crystallites co-exist in the films. However, the intensity of the $(h 00)$ family peaks increases significantly upon doping. This is indicative of an increase in relative crystallinity induced by the dopants, and consistent with previous literature for doped P(NDI20D-T2). No diffraction peaks related to the dopant phase are ob- served, which is indicative of either a good polymerdopant intermixing or an inability of the dopant to crystallize at this concentration (15 mol\%). This is in agreement with atomic force microscope analysis that show no visible aggregates regardless of the dopant used (Figure S24). Figure S25a presents a zoom in the in-plane (100) peak, the integrated area of which can serve as a measure of crystallinity. A relative crystallinity has been estimated by normalizing the area of the (100) peak of the doped samples with respect to that of the pristine one and is presented in Figure S25b. A linear increase of crystallinity is observed as the energy of the SOMO level decreases, with the exception of the outlier $\mathbf{1 H}$. Besides a crystallinity increase, doping induces a shift of the (100) peak along the $\mathrm{q}_{\mathrm{z}}$ axis towards higher values (Figure S26a), which is indicative of a reduced out-of-plane $d_{100}$ spacing (Figure S26b). Note that no apparent shift is visible along the in-plane direction, implying that only the edge-on oriented crystallites are affected by dopants and not the face-on ones. No other unit cell parameters are affected by doping, suggesting that the dopants are intercalated between the in-plane lamellae. Intuitively, the increased crystallinity (Figure S25b) and decreased lamellar spacing (Figure S26b) for higherlying SOMO levels can easily be correlated with the increase in conductivity in Figure $4 \mathrm{~b}$. The prerequisite for such a causal relation to hold is that the long-range charge transport is limited by the intra-grain instead of intergrain transport, which is not upfront evident in polycrystalline films. In fact, it is known that interconnectivity between grains is more important than crystallinity in this class of donor-acceptor polymers. ${ }^{62 *}$ Moreover, several factors point towards a more complicated situation. First, the outliers at SOMO energies of $-3.02 \mathrm{eV}$ and $-2.99 \mathrm{eV}$ in Figure S25b and S26b, respectively, do not correlate with any noticeable features in the conductivity trend. Moreover, while better molecular packing logically leads to larger intra-grain transfer integrals and higher inter-grain mobilities, it is harder to reconcile with the exponential increase in charge density, c.f. Figure 5, and the concomitant decrease in magnitude of the Seebeck coefficient (Figure 4d). Note that neither charge density nor Seebeck coefficient shows any deviations from smooth trends at the outliers in terms of packing that are mentioned above. Thus, we mainly attributed the variations in conductivity to the effect of SOMO energy.

Analytical conductivity modeling. In order to investigate the potential role of the energetics, and particularly the position of the SOMO with respect to the P(NDI2ODT2) LUMO level, we analytically modeled the conductivity of the two-compound (semiconductor and dopant) system using a variable range hopping (VRH) model. We deliberately did not include any morphology or packing aspects as to focus on the energetics only. The model has previously been used to describe the conductivity and thermopower of doped organic semiconductors. ${ }^{63-65}$

Using Miller-Abrahams hopping rates, the conductivity is assumed to be dominated by a characteristic hop over a distance $R^{*}$ and going from the Fermi energy $E_{F}$ to an energy $E^{*}$ as:

$$
\sigma=\sigma_{0} \exp \left(-2 \alpha R^{*}-\frac{E^{*}-E_{F}}{k_{B} T}\right)
$$


where $\sigma_{0}$ is a conductivity prefactor, $\sigma_{0}=n \mu_{0}=$ $N_{0} \frac{R^{* 2} v_{0}}{p \sigma_{0, D O S}}$. Here, $n$ is the charge carrier density that is set equal to the density of active dopants $N_{D, a c t}$, i.e. the density of dopants that undergo integer charge transfer and contribute a free charge to the system, $\sigma_{0, D O S}$ is the width of the Gaussian DOS $g_{0}(E)=\frac{N_{0}}{\sqrt{2 \pi \sigma_{0, D O S}^{2}}} \exp \left(\frac{-\left(E-E_{0}\right)^{2}}{2 \sigma_{0, D O S}^{2}}\right)$ of the pristine (undoped) material and $N_{0}$ is the total site density. The characteristic hopping distance $R^{*}$ and the critical or transport energy $E^{*}$ are connected to each other through the density of states $g(E)$ :

$$
B_{C}=\frac{4}{3} \pi R^{* 3} \int_{E_{F}}^{E^{*}} g(E) d E
$$

where $B_{C} \approx 2.7$ is the critical number of bonds on the percolating network.

It is well established that the ionized dopants induce a deep tail in the original DOS. Here, we follow Ref. ${ }^{63}$ who extended the work by Arkhipov et al. ${ }^{66}$ to systems with nonzero energy differences between the relevant level in the dopant and the semiconductor, here the SOMO and LUMO energies, respectively. Defining $E_{C}\left(r=N_{i}^{-1 / 3}\right)=-q^{2} / 4 \pi \varepsilon_{0} \varepsilon_{r} r$ as the Coulomb energy a typical inter-site distance away from the ionized dopant and $A=\frac{4 \pi q^{6} N_{d}}{\left(4 \pi \varepsilon_{0} \varepsilon_{r}\right)^{3}}, g(E)$ becomes

$$
\begin{gathered}
g(E)=\left(1-\frac{4 \pi N_{d}}{3 N_{i}}\right) \frac{g_{1}(E)}{\int_{-\infty}^{0} d E g_{1}(E)}+\frac{4 \pi N_{d}}{3 N_{i}} \frac{g_{2}(E)}{\int_{-\infty}^{0} d E g_{2}(E)} \\
g_{1}(E)=A \int_{E_{1}}^{0} \frac{d E_{c}}{E_{C}^{4}} \exp \left(\frac{A}{3 E_{C}^{3}}\right) g_{0}\left(E-E_{C}\right) \\
g_{2}(E)=A \int_{-\infty}^{E_{1}} \frac{d E_{c}}{E_{C}^{4}} \exp \left(\frac{A}{3 E_{C}^{3}}\right) g_{0}\left(E-\Delta E-E_{C}\right) .
\end{gathered}
$$

In this framework, the Seebeck coefficient can be well approximated by: 65

$$
S \cong-\frac{E^{*}-E_{F}}{q T} .
$$

Typical results of applying the VRH model to calculate conductivity and thermopower as a function of $\Delta E=$ $E_{\text {Sомо }}-E_{L U M O}$, the energy difference between the dopant SOMO and semiconductor LUMO, are shown in Figure 7. The parameters used are typical for this type of compounds ${ }^{64}$ and the shown trends are not sensitive to the particular values used. Importantly, the key observations in Figure 4 are reproduced by the VRH model for intermediate charge concentrations $n / N_{0}$ of $1-3 \%$, consistent with the limited doping efficiency experimentally observed for this polymer/dopant system. ${ }^{29}$ In particular, the exponential dependence of conductivity on dopant SOMO with a leveling off towards deeper SOMO energies (more negative $\Delta E$ ) is quasi-quantitatively reproduced by the model.

At larger positive $\Delta E$, the modeled conductivity also levels off due to all dopants having contributed a 'free' charge to the system, i.e. having been ionized; note that these charges still feel the long-range Coulomb attraction from the dopant ions. The exponential trend of the conductivity at intermediate $\Delta E$ reflects the thermal activation of charge carriers out of the trap formed by the Coulomb po- tential and, if $\Delta E<0$, the energy offset towards the transport energy $E^{*}$ that sits on the host LUMO. At large negative $\Delta E$ this activation becomes increasingly difficult and conductivity via direct hopping between dopants becomes dominant. In other words, $E^{*}$ shifts from the host LUMO to the guest SOMO. The associated drop in $E^{*}-E_{F}$ is clearly visible as a relatively sudden shift towards less negative Seebeck coefficients in panel b. Interestingly, the experimental thermopower data in Figure $4 d$ seem to qualitatively show the same behavior. Finally, also the trend in the measured work function (Figure S21) is reproduced in the simulated Fermi energy in the inset of Figure 7. Summarizing, the simulation results seem to strongly indicate that for the investigated system, the charge and energy transport are dominated by the (relative) position of the SOMO level, whereas the observed morphological differences appear to play a lesser role.
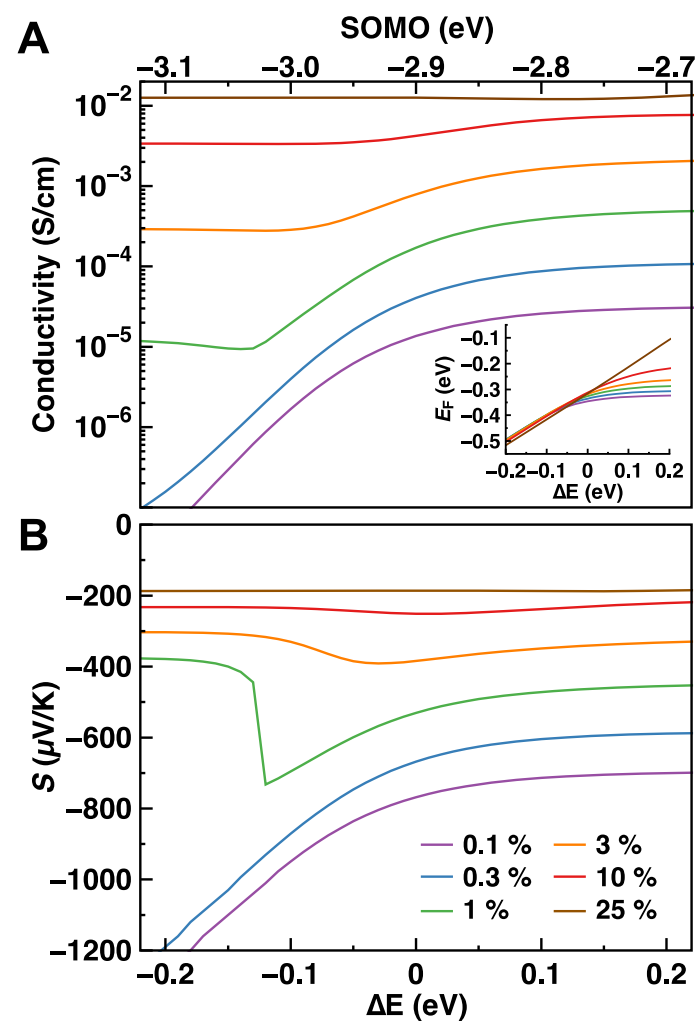

Figure 7. Calculated conductivity (a) and thermopower (b) vs. SOMO - LUMO energy difference for different relative charge carrier concentrations $n / N_{0}$ as indicated in the legend. The top axis indicates a hypothetical SOMO energy for comparison with Figure 4, calculated assuming $E_{L U M O}=-3.9 \mathrm{eV}$ and an empirical $1 \mathrm{eV}$ offset to account for systematic errors in e.g. the absolute SOMO positions as obtained from DFT. The inset is the corresponding position of the Fermi level w.r.t. the center of the pristine LUMO. Other parameters used are $\varepsilon_{r}=3.6$, $N_{0}=6.4 \times 10^{25} \mathrm{~m}^{-3}, v_{0}=3.9 \times 10^{10} \mathrm{~s}^{-1}, \sigma_{0, D O S}=0.075 \mathrm{eV}, \alpha=$ $7.1 \times 10^{8} \mathrm{~m}^{-1}$.

\section{CONCLUSIONS}

In conclusion, we investigated the impact of SOMO energy on the n-doping efficiency of benzimidazole-based dopants. By designing and synthesizing a series of new airstable benzimidazole-based dopants with different SOMO 
energy levels, we demonstrated that a reduction of the dopant SOMO energy by only $\sim 0.3 \mathrm{eV}$ (i.e., less negative SOMO) boosts the electrical conductivity by more than one order of magnitude. This is corroborated by a reduction of Seebeck coefficient, which is indicative of a larger carrier concentration when high-lying SOMO dopants are used as the reducing agents. Quantitative EPR analysis reveals that the spin density increases exponentially with higher lying SOMO energy, an observation which is corroborated by Kelvin probe and in agreement with the electrical measurements. The solid-state microstructural changes observed with GIWAXS are hard to reconcile with the observed exponential increase in charge density and electrical conductivity, as well as the concomitant decrease in magnitude of the Seebeck coefficient. Thus, we mainly attributed the variations in conductivity to the effect of SOMO energy. This is supported by an analytical model which strongly indicates that charge and energy transport are dominated by the (relative) position of the SOMO level, whereas the observed morphological differences appear to play a lesser role. These results set molecular-design guidelines for next-generation n-type dopants.

\section{ASSOCIATED CONTENT}

\section{Supporting Information}

The Supporting Information is available free of charge on the ACS Publications website at DOI: 10.1021/acs.chemmater.XXXX.

Synthetic procedures and additional characterization data, such as ${ }^{1} \mathrm{H}-\mathrm{NMR}$, DSC, TGA, CV, DPV, KP, UV-vis, AFM, GIWAXS (PDF).

\section{AUTHOR INFORMATION}

\section{Corresponding Author}

*E-mail: gabriele.dicarlo@unimi.it (G.D.C.), simone.fabiano@liu.se (S.F.).

\section{Author Contributions}

The manuscript was written through contributions of all authors. All authors have given approval to the final version of the manuscript.

\section{Notes}

The authors declare no competing financial interest.

\section{ACKNOWLEDGMENT}

The authors acknowledge the Swedish Foundation for Strategic Research, VINNOVA (2015-04859), the Swedish Research Council (2016-03979), ÅForsk (18-313) and the Advanced Functional Materials Center at Linköping University (200900971). GIWAXS experiments were performed at the NCDSWEET beamline of ALBA synchrotron with the collaboration of ALBA staff. Regione Lombardia and Fondazione Cariplo are gratefully acknowledged for financial support and for the use of instrumentations purchased through the SmartMatLab Centre project (2014-42639194). The authors acknowledge Serena Cappelli for DSC and TGA analysis. G.D.C. greatly thanks the Università degli Studi di Milano (Piano Sostegno alla Ricerca 2018 LINEA 2 Azione A - Giovani Ricercatori) for financial support.

\section{REFERENCES}

(1) Root, S. E.; Savagatrup, S.; Printz, A. D.; Rodriquez, D.; Lipomi, D. J. Mechanical Properties of Organic Semiconductors for Stretchable, Highly Flexible, and Mechanically Robust Electronics. Chemical Reviews 2017, 117 (9), 6467-6499.

(2) Facchetti, A. $\pi$-Conjugated Polymers for Organic Electronics and Photovoltaic Cell Applications. Chemistry of Materials 2011, 23 (3), 733-758.

(3) Sekine, C.; Tsubata, Y.; Yamada, T.; Kitano, M.; Doi, S. Recent Progress of High Performance Polymer OLED and OPV Materials for Organic Printed Electronics. Science and Technology of Advanced Materials 2014, 15 (3), 034203.

(4) Li, N.; Baran, D.; Spyropoulos, G. D.; Zhang, H.; Berny, S.; Turbiez, M.; Ameri, T.; Krebs, F. C.; Brabec, C. J. Environmentally Printing Efficient Organic Tandem Solar Cells with High Fill Factors: A Guideline towards 20\% Power Conversion Efficiency. Advanced Energy Materials 2014, 4 (11), 1400084.

(5) McGrail, B. T.; Sehirlioglu, A.; Pentzer, E. Polymer Composites for Thermoelectric Applications. Angewandte Chemie International Edition 2015, 54 (6), 1710-1723.

(6) Chen, Y.; Zhao, Y.; Liang, Z. Solution Processed Organic Thermoelectrics: Towards Flexible Thermoelectric Modules. Energy and Environmental Science 2015, 8 (2), 401-422.

(7) Petsagkourakis, I.; Tybrandt, K.; Crispin, X.; Ohkubo, I.; Satoh, N.; Mori, T. Thermoelectric Materials and Applications for Energy Harvesting Power Generation. Science and Technology of Advanced Materials 2018, 19 (1), 836-862.

Beretta, D.; Neophytou, N.; Hodges, J. M.; Kanatzidis, M. G.; Narducci, D.; Martin-Gonzalez, M.; Beekman, M.; Balke, B.; Cerretti, G.; Tremel, W.; et al. Thermoelectrics: From History, a Window to the Future. Materials Science and Engineering R: Reports 2018.

(9) Kroon, R.; Mengistie, D. A.; Kiefer, D.; Hynynen, J.; Ryan, J. D.; Yu, L.; Müller, C. Thermoelectric Plastics: From Design to Synthesis, Processing and Structure-Property Relationships. Chemical Society Reviews 2016, 45 (22), 6147-6164.

(10) Meng, Q.; Cai, K.; Chen, Y.; Chen, L. Research Progress on Conducting Polymer Based Supercapacitor Electrode Materials. Nano Energy 2017, 36, 268-285.

(11) Katz, H. E.; Searson, P. C.; Poehler, T. O. Batteries and Charge Storage Devices Based on Electronically Conducting Polymers. Journal of Materials Research 2010, 25 (8), 15611574.

(12) Bubnova, O.; Crispin, X. Towards Polymer-Based Organic Thermoelectric Generators. Energy and Environmental Science 2012, 5 (11), 9345-9362.

(13) Fabretto, M.; Jariego-Moncunill, C.; Autere, J. P.; Michelmore, A.; Short, R. D.; Murphy, P. High Conductivity PEDOT Resulting from Glycol/Oxidant Complex and Glycol/Polymer Intercalation during Vacuum Vapour Phase Polymerisation. Polymer 2011, 52 (8), 1725-1730.

(14) Kao, C. Y.; Lee, B.; Wielunski, L. S.; Heeney, M.; McCulloch, I.; Garfunkel, E.; Feldman, L. C.; Podzorov, V. Doping of Conjugated Polythiophenes with Alkyl Silanes. Advanced Functional Materials 2009, 19 (12), 1906-1911.

(15) Kim, N.; Kee, S.; Lee, S. H.; Lee, B. H.; Kahng, Y. H.; Jo, Y. R.; Kim, B. J.; Lee, K. Highly Conductive PEDOT:PSS Nanofibrils Induced by Solution-Processed Crystallization. Advanced Materials 2014, 26 (14), 2268-2272.

(16) Shi, K.; Zhang, F.; Di, C. A.; Yan, T. W.; Zou, Y.; Zhou, X.; Zhu, D.; Wang, J. Y.; Pei, J. Toward High Performance N-Type Thermoelectric Materials by Rational Modification of BDPPV Backbones. Journal of the American Chemical Society 2015, 137 (22), 6979-6982.

(17) Wang, S.; Sun, H.; Erdmann, T.; Wang, G.; Fazzi, D.; Lappan, U.; Puttisong, Y.; Chen, Z.; Berggren, M.; Crispin, X.; et al. A Chemically Doped Naphthalenediimide-Bithiazole Polymer for n-Type Organic Thermoelectrics. Advanced Materials 2018, 30 (31), 1801898.

(18) Zhao, X.; Madan, D.; Cheng, Y.; Zhou, J.; Li, H.; Thon, S. M.; Bragg, A. E.; DeCoster, M. E.; Hopkins, P. E.; Katz, H. E. High Conductivity and Electron-Transfer Validation in an n-Type 
Fluoride-Anion-Doped Polymer for Thermoelectrics in Air. Advanced Materials 2017, 29 (34), 1606928.

(19) Ma, W.; Shi, K.; Wu, Y.; Lu, Z. Y.; Liu, H. Y.; Wang, J. Y.; Pei, J. Enhanced Molecular Packing of a Conjugated Polymer with High Organic Thermoelectric Power Factor. ACS Applied Materials and Interfaces 2016, 8 (37), 24737-24743.

(20) Kao, P. C.; Wang, J. Y.; Lin, J. H.; Yang, C. H. Effects of the Na 2 CO 3 Dopant on Electron Injection and Transport in Organic Light Emitting Devices. Thin Solid Films 2013, 527, 338-343.

(21) Barbot, A.; Lucas, B.; Di Bin, C.; Ratier, B. Cesium CarbonateDoped 1,4,5,8-Naphthalene-Tetracarboxylic-Dianhydride Used as Efficient Electron Transport Material in Polymer Solar Cells. Organic Electronics: physics, materials, applications 2014, 15 (4), 858-863.

(22) Ali Benamara, A.; Galtier, M.; Montaner, A. N Doping of Polyacetylene. Synthetic Metals 1991, 41 (1-2), 45-48.

(23) Parthasarathy, G.; Shen, C.; Kahn, A.; Forrest, S. R. Lithium Doping of Semiconducting Organic Charge Transport Materials. Journal of Applied Physics 2001, 89 (9), 49864992.

(24) Chiang, C. K.; Gau, S. C.; Fincher, C. R.; Park, Y. W.; MacDiarmid, A. G.; Heeger, A. J. Polyacetylene, (CH)x: N-Type and p-Type Doping and Compensation. Applied Physics Letters 1978, 33 (1), 18-20.

(25) Goetz, K. P.; Vermeulen, D.; Payne, M. E.; Kloc, C.; McNeil, L. E.; Jurchescu, O. D. Charge-Transfer Complexes: New Perspectives on an Old Class of Compounds. Journal of Materials Chemistry C 2014, 2 (17), 3065-3076.

(26) Menke, T.; Wei, P.; Ray, D.; Kleemann, H.; Naab, B. D.; Bao, Z.; Leo, K.; Riede, M. A Comparison of Two Air-Stable Molecular n-Dopants for C60. Organic Electronics: physics, materials, applications 2012, 13 (12), 3319-3325.

(27) Wei, P.; Menke, T.; Naab, B. D.; Leo, K.; Riede, M.; Bao, Z. 2-(2Methoxyphenyl)-1,3-Dimethyl-1 H-Benzoimidazol-3-Ium Iodide as a New Air-Stable n-Type Dopant for VacuumProcessed Organic Semiconductor Thin Films. Journal of the American Chemical Society 2012, 134 (9), 3999-4002.

(28) Zhu, X.-Q.; Zhang, M.-T.; Yu, A.; Wang, C.-H.; Cheng, J.-P. Hydride, Hydrogen Atom, Proton, and Electron Transfer Driving Forces of Various Five-Membered Heterocyclic Organic Hydrides and Their Reaction Intermediates in Acetonitrile. Journal of the American Chemical Society 2008 130 (8), 2501-2516.

(29) Schlitz, R. A.; Brunetti, F. G.; Glaudell, A. M.; Miller, P. L.; Brady, M. A.; Takacs, C. J.; Hawker, C. J.; Chabinyc, M. L. Solubility-Limited Extrinsic n-Type Doping of a High Electron Mobility Polymer for Thermoelectric Applications. Advanced Materials 2014, 26 (18), 2825-2830.

(30) Wei, P.; Oh, J. H.; Dong, G.; Bao, Z. Use of a $1 \mathrm{H}-$ Benzoimidazole Derivative as an n-Type Dopant and To Enable Air-Stable Solution-Processed n-Channel Organic Thin-Film Transistors. Journal of American Chemical Society 2010, 132, 8852-8853.

(31) Naab, B. D.; Guo, S.; Olthof, S.; Evans, E. G. B.; Wei, P.; Millhauser, G. L.; Kahn, A.; Barlow, S.; Marder, S. R.; Bao, Z. Mechanistic Study on the Solution-Phase n-Doping of 1,3Dimethyl-2-Aryl-2, 3-Dihydro-1H-Benzoimidazole Derivatives. Journal of the American Chemical Society 2013, 135 (40), 15018-15025.

(32) Denti, I.; Cimò, S.; Brambilla, L.; Milani, A.; Bertarelli, C.; Tommasini, M.; Castiglioni, C. Polaron Confinement in $\mathrm{N}$ Doped P(NDI20D-T2) Unveiled by Vibrational Spectroscopy. Chemistry of Materials 2019.

(33) Bin, Z.; Liu, Z.; Duan, L. Organic Radicals Outperform LiF as Efficient Electron-Injection Materials for Organic LightEmitting Diodes. Journal of Physical Chemistry Letters 2017, 8 (19), 4769-4773.

(34) Wei, P.; Liu, N.; Lee, H. R.; Adijanto, E.; Ci, L.; Naab, B. D.; Zhong, J. Q.; Park, J.; Chen, W.; Cui, Y.; et al. Tuning the Dirac Point in CVD-Grown Graphene through Solution Processed n-Type Doping with 2-(2-Methoxyphenyl)-1,3-Dimethyl-2,3Dihydro-1 H-Benzoimidazole. Nano Letters 2013, 13 (5), 1890-1897.

(35) Wang, S.; Sun, H.; Ail, U.; Vagin, M.; Persson, P. O. Å.
Andreasen, J. W:; Thiel, W.; Berggren, M.; Crispin, X.; Fazzi, D.; et al. Thermoelectric Properties of Solution-Processed nDoped Ladder-Type Conducting Polymers. Advanced Materials 2016, 28 (48), 10764-10771.

(36) Saglio, B.; Mura, M.; Massetti, M.; Scuratti, F.; Beretta, D.; Jiao, X.; McNeill, C. R.; Sommer, M.; Famulari, A.; Lanzani, G.; et al. $\mathrm{N}$-Alkyl Substituted $1 \mathrm{H}$-Benzimidazoles as Improved $\mathrm{n}$ Type Dopants for a Naphthalene-Diimide Based Copolymer. Journal of Materials Chemistry A 2018, 6 (31), 15294-15302.

(37) Kiefer, D.; Giovannitti, A.; Sun, H.; Biskup, T.; Hofmann, A.; Koopmans, M.; Cendra, C.; Weber, S.; Koster, L. J. A.; Olsson, E.; et al. Enhanced N-Doping Efficiency of a Naphthalenediimide-Based Copolymer through Polar Side Chains for Organic Thermoelectrics. ACS Energy Letters 2018, 3 (2), 278-285.

(38) Shin, Y.; Massetti, M.; Komber, H.; Biskup, T.; Nava, D.; Lanzani, G.; Caironi, M.; Sommer, M. Improving Miscibility of a Naphthalene Diimide-Bithiophene Copolymer with n-Type Dopants through the Incorporation of "Kinked" Monomers. Advanced Electronic Materials 2018, 4 (10), 1700581.

(39) Liu, J.; Qiu, L.; Alessandri, R.; Qiu, X.; Portale, G.; Dong, J. J.; Talsma, W.; Ye, G.; Sengrian, A. A.; Souza, P. C. T.; et al. Enhancing Molecular N-Type Doping of Donor-Acceptor Copolymers by Tailoring Side Chains. Advanced Materials 2018, 30 (7), 1704630.

(40) Wang, Y.; Nakano, M.; Michinobu, T.; Kiyota, Y.; Mori, T.; Takimiya, K. NaphthodithiophenediimideBenzobisthiadiazole-Based Polymers: Versatile n-Type Materials for Field-Effect Transistors and Thermoelectric Devices. Macromolecules 2017, 50 (3), 857-864.

(41) Liu, J.; Shi, Y.; Dong, J.; Nugraha, M. I.; Qiu, X.; Su, M.; Chiechi, R. C.; Baran, D.; Portale, G.; Guo, X.; et al. Overcoming Coulomb Interaction Improves Free-Charge Generation and Thermoelectric Properties for n-Doped Conjugated Polymers. ACS Energy Letters 2019, 1556-1564.

(42) Liu, J.; Qiu, L.; Portale, G.; Koopmans, M.; ten Brink, G.; Hummelen, J. C.; Koster, L. J. A. N-Type Organic Thermoelectrics: Improved Power Factor by Tailoring HostDopant Miscibility. Advanced Materials 2017, 29 (36), 1701641

(43) Bard, A. J.; Faulkner, L. R. Electrochemical Methods Fundamentals and Applications; Wiley, New York, 2002.

Gritzner, G.; Kuta, J. Recommendations on Reporting Electrode Potentials in Nonaqueous Solvents. Pure \& Appil. Chem. 1984, 56, 461-466.

(45) Gritzner, G. Polarographic Half-Wave Potentials of Cations in Nonaqueous Solvents. Pure \& Appil. Chem. 1990, 62 (9), 1839-1858.

(46) Renaud, G.; Lazzari, R.; Leroy, F. Probing Surface and Interface Morphology with Grazing Incidence Small Angle XRay Scattering. Surface Science Reports 2009, 64 (8), 255380

(47) Frisch, M. J.; Trucks, G. W.; Schlegel, H. B.; Scuseria, G. E.; Robb, M. A.; Cheeseman, J. R.; Scalmani, G.; Barone, V.; Petersson, G. A.; Nakatsuji, H.; et al. Gaussian 16. Gaussian, Inc., Wallingford 2016

(48) Scalmani, G.; Frisch, M. J. Continuous Surface Charge Polarizable Continuum Models of Solvation. I. Genera Formalism. Journal of Chemical Physics 2010, 132, 114110.

(49) Zhu, C.; Wei, Y. An Inorganic Iodine-Catalyzed Oxidative System for the Synthesis of Benzimidazoles Using Hydrogen Peroxide under Ambient Conditions. ChemSusChem 2011, 4 (8), 1082-1086.

(50) Chen, L.; Qu, X. Synthesis, Characterization and Crystal Structure of Iridium(III)Bis(2-p- Tolyl-Benzothiazolato$\mathrm{N}, \mathrm{C} 2)$ (Acetylacetonate). Journal of Chemical Crystallography 2009, 39 (4), 241-245.

(51) Chen, L.; Yang, C.; Li, S.; Qin, J. Synthesis and Electronic Absorption and Fluorescence of 2-Arylbenzothiazole Derivatives. Spectrochimica Acta - Part A 2007, 68 (2), 317322.

(52) Lou, A. J. T.; Righetto, S.; Barger, C.; Zuccaccia, C.; Cariati, E.; Macchioni, A.; Marks, T. J. Unprecedented Large Hyperpolarizability of Twisted Chromophores in Polar 
Media. Journal of the American Chemical Society 2018, 140 (28), 8746-8755.

(53) Lee, I.-S. H.; Kil, H. J.; Ji, Y. R. Reactivities of Acridine Compounds in Hydride Transfer Reactions. Journal of Physical Organic Chemistry 2007, 20, 484-490.

(54) Wang, S.; Fazzi, D.; Puttisong, Y.; Jafari, M. J.; Chen, Z.; Ederth, T.; Andreasen, J. W.; Chen, W. M.; Facchetti, A.; Fabiano, S. Effect of Backbone Regiochemistry on Conductivity, Charge Density, and Polaron Structure of n-Doped Donor-Acceptor Polymers. Chemistry of Materials 2019, 31, 3395-3406.

(55) Huang, D.; Yao, H.; Cui, Y.; Zou, Y.; Zhang, F.; Wang, C.; Shen H.; Jin, W.; Zhu, J.; Diao, Y.; et al. Conjugated-Backbone Effect of Organic Small Molecules for n-Type Thermoelectric Materials with ZT over 0.2. Journal of the American Chemical Society 2017, 139 (37), 13013-13023.

(56) Menke, T.; Ray, D.; Meiss, J.; Leo, K.; Riede, M. In-Situ Conductivity and Seebeck Measurements of Highly Efficient n-Dopants in Fullerene C 60. Applied Physics Letters 2012 , 100 (9), 093304.

(57) Euvrard, J.; Revaux, A.; Bayle, P. A.; Bardet, M.; Vuillaume, D.; Kahn, A. The Formation of Polymer-Dopant Aggregates as a Possible Origin of Limited Doping Efficiency at High Dopant Concentration. Organic Electronics: physics, materials, applications 2018, 53, 135-140.

(58) Naab, B. D.; Gu, X.; Kurosawa, T.; To, J. W. F.; Salleo, A.; Bao, Z Role of Polymer Structure on the Conductivity of N-Doped Polymers. Advanced Electronic Materials 2016, 2 (5), 1600004.

(59) Rivnay, J.; Toney, M. F.; Zheng, Y.; Kauvar, I. V.; Chen, Z.; Wagner, V.; Facchetti, A.; Salleo, A. Unconventional Face-on Texture and Exceptional in-Plane Order of a High Mobility nType Polymer. Advanced Materials 2010, 22 (39), 43594363.

(60) Steyrleuthner, R.; Di Pietro, R.; Collins, B. A.; Polzer, F.; Himmelberger, S.; Schubert, M.; Chen, Z.; Zhang, S.; Salleo, A.; Ade, H.; et al. The Role of Regioregularity, Crystallinity, and Chain Orientation on Electron Transport in a High-Mobility n-Type Copolymer. Journal of the American Chemical Society 2014, 136 (11), 4245-4256.

(61) Rivnay, J.; Steyrleuthner, R.; Jimison, L. H.; Casadei, A.; Chen, Z.; Toney, M. F.; Facchetti, A.; Neher, D.; Salleo, A. Drastic Control of Texture in a High Performance N-Type Polymeric Semiconductor and Implications for Charge Transport. Macromolecules 2011, 44 (13), 5246-5255.

(62) Wang, S.; Fabiano, S.; Himmelberger, S.; Puzinas, S.; Crispin X.; Salleo, A.; Berggren, M. Experimental Evidence That Short-Range Intermolecular Aggregation Is Sufficient for Efficient Charge Transport in Conjugated Polymers. Proceedings of the National Academy of Sciences 2015, 112 (34), 10599-10604.

(63) Zuo, G.; Abdalla, H.; Kemerink, M. Impact of Doping on the Density of States and the Mobility in Organic Semiconductors. Physical Review B 2016, 93 (23), 235203.

(64) Abdalla, H.; Zuo, G.; Kemerink, M. Range and Energetics of Charge Hopping in Organic Semiconductors. Physical Review $B$ 2017, 96 (24), 241202.

(65) Zuo, G.; Abdalla, H.; Kemerink, M. Conjugated Polymer Blends for Organic Thermoelectrics. Advanced Electronic Materials 2019, 1800821.

(66) Arkhipov, V. I.; Heremans, P.; Emelianova, E. V.; Bässler, H. Effect of Doping on the Density-of-States Distribution and Carrier Hopping in Disordered Organic Semiconductors. Physical Review B 2005, 71 (4), 045214. 
ToC

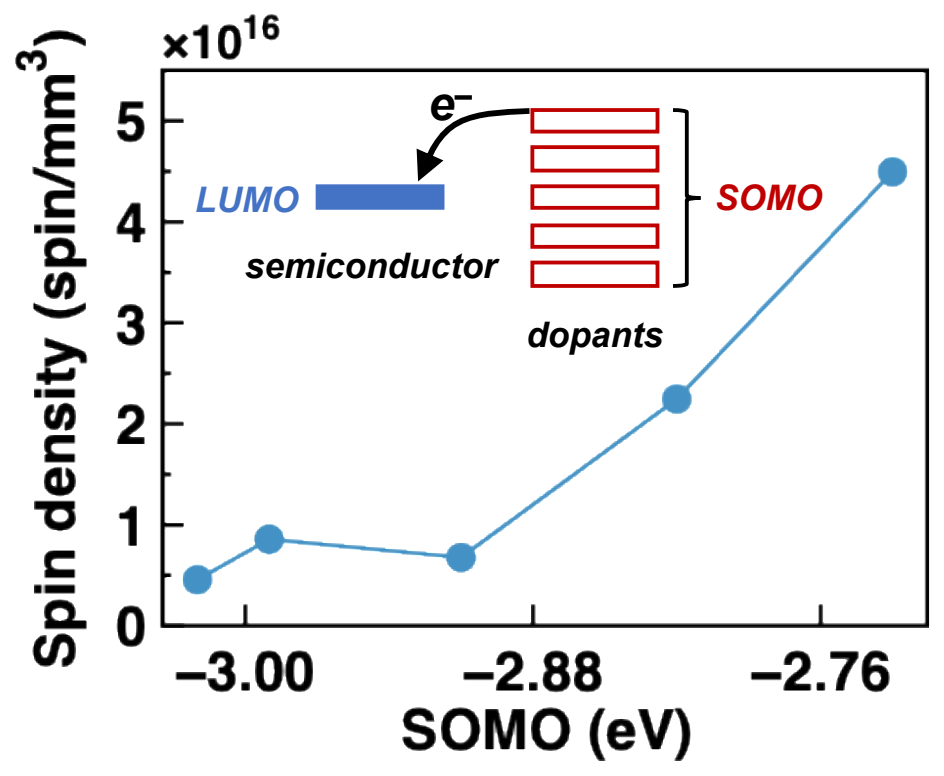

